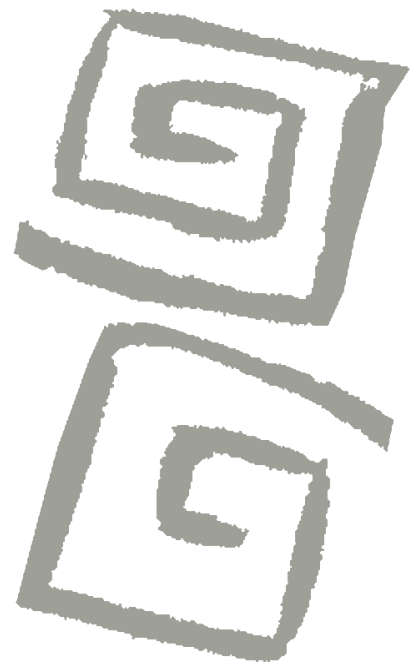

'Médico Cirujano, Magíster en Gestión y Políticas Públicas. Estudiante de Posgrado, Facultad de Medicina, Universidad de Buenos Aires. Buenos Aires, Argentina. $\triangle$ (iD)

${ }^{2}$ Cirujano Dentista. Asistente de Investigación, Instituto de Investigación, Facultad de Odontologia, Universidad Católica los Ángeles de Chimbote. Chimbote, Perú. $\triangle$ iD

${ }^{3}$ Licenciada en Enfermería, Especialista en Cuidados Intensivos. Enfermera asistencial, Instituto Nacional Cardiovascular (INCOR), EsSalud. Lima, Perú. $\bowtie$ iD

${ }^{4}$ Médico Cirujano, Asesor científico, Sociedad Científica de Estudiantes de Medicina de Ica (SOCEMI), Facultad de Medicina, Universidad Nacional San Luis Gonzaga. Ica, Perú. $\triangle$ iD

${ }^{5}$ Médico Cirujano. Editor adjunto, Oficina General de Información y Sistemas, Instituto Nacional de Salud. Lima, Perú. $\square$ iD

${ }^{6}$ Médico Cirujano, Magíster en Salud Pública. Asesor, Sociedad Científica de Estudiantes de Medicina de la Amazonía Peruana Facultad de Medicina, Universidad Nacional de la Amazonía Peruana. Iquitos, Perú. $\bowtie$ iD

TOdontólogo. Doctor en Salud Pública. Jefe del Centro de Investigación y Extensión, Facultad de Odontología, Universidad de Antioquia. Medellín, Colombia. $\triangle$ iD

\section{Acceso a servicios de salud dental en menores de doce años en Perú, 2014}

\author{
Access to oral health services in children under twelve \\ years of age in Peru, 2014
}

\begin{abstract}
Akram Hernández-Vásquez¹, Diego Azañedo², Deysi Díaz-Seijas³, Guido Bendezú-Quispe ${ }^{4}$, Hugo Arroyo-Hernández ${ }^{5}$, Stalin Vilcarromero ${ }^{6}$, Andrés A. Agudelo-Suárez ${ }^{7}$
\end{abstract}

RESUMEN El objetivo del estudio fue explorar los patrones de acceso a servicios de salud dental en menores de doce años en Perú. Se revisaron datos de 25.285 niños menores de 12 años que participaron en la Encuesta Demográfica y de Salud Familiar 2014. Se realizó un análisis exploratorio espacial para proyectar las proporciones de menores con acceso a servicios de salud dental según regiones a nivel nacional, tipo de servicio de salud y lugar de residencia urbana o rural. Los resultados muestran que el $26,7 \%$ de la muestra tuvo acceso a los servicios de salud dental en los últimos seis meses. El $39,6 \%$ pertenecía al grupo etario de 0 a 4 años, el 40,6\% habitaba en la zona andina y un 58,3\% residía en zonas urbanas. Las regiones de Huancavelica, Apurímac, Ayacucho, Lima y Pasco tuvieron los mayores porcentajes de población que accedió a servicios de salud dental a nivel nacional. En conclusión, existe un bajo acceso a los servicios de salud dental para la población menor de 12 años en Perú. La distribución espacial del acceso a los servicios de salud dental permitiría identificar y agrupar regiones según patrones comunes de acceso para enfocar acciones en materia de salud pública.

PALABRAS CLAVES Sistemas de Información Geográfica; Accesibilidad a los Servicios de Salud; Salud Bucal; Atención Dental para Niños; Perú.

ABSTRACT The aim of the study was to explore the patterns of dental health services access in children under twelve years of age in Peru. Data from 25,285 children under 12 years who participated in the Demographic and Family Health Survey of 2014 were reviewed. An exploratory spatial analysis was performed to project the proportions of children with access to dental health services, according to national regions, type of health service and urban or rural place of residence. The results show that of the total sample, $26.7 \%$ had access to dental health services in the last six months, $39.6 \%$ belonged to the age group 0-4 years, 40.6\% lived in the Andean region and 58.3\% lived in urban areas. The regions of Huancavelica, Apurimac, Ayacucho, Lima and Pasco had the highest percentages of access nationwide. In conclusion, there is low access to dental health services in the population under 12 years of age in Peru. The spatial distribution of access to dental health services allows regions to be identified and grouped according to similar access patterns, in order to better focus public health actions.

KEY WORDS Geographic Information Systems; Health Services Accessibility; Oral Health; Dental Care for Children; Peru. 


\section{INTRODUCCIÓN}

La caries dental es la patología más prevalente entre las enfermedades orales y es muy frecuente en la edad pediátrica ${ }^{(1)}$. El no tratamiento de esta y otras afecciones bucales puede tener consecuencias físicas, psicológicas, nutricionales, económicas e incluso sobre la salud sistémica de la persona que las padece ${ }^{(2,3)}$. En el Perú, la prevalencia de caries dental se ha estimado en el 90,4\% de la población escolar menor de 15 años, cifra que ubica al país en una posición desfavorecida con respecto a la salud oral en niños de países vecinos como Venezuela y Colombia ${ }^{(4,5)}$. La Academia Americana de Odontología Pediátrica (AAPD, por sus siglas en inglés), recomienda realizar por lo menos una visita al odontólogo cada seis meses para mantener una buena salud oral a través de exámenes y métodos preventivos ${ }^{(6)}$. Sin embargo, dichas visitas deberían basarse en el riesgo de caries individual del niño, llegando a ser visitas mensuales para niños con alto riesgo ${ }^{(7)}$.

A pesar de que a lo largo de los años los organismos de salud internacionales han recomendado la prevención de la caries dental como la mejor estrategia para reducir su prevalencia, la producción científica en salud oral a nivel mundial muestra escasa atención a temas de salud pública desde un enfoque preventivo-promocional y una mayor producción enfocada a la odontología restauradora ${ }^{(8,9)}$.

En Perú, desde el año 2013, el Instituto Nacional de Estadística e Informática (INEI) incluyó dentro del cuestionario de salud de la Encuesta Demográfica y de Salud Familiar (ENDES) ciertos aspectos relacionados con la salud oral. Uno de ellos es el acceso a la atención en servicios odontológicos para ciertas etapas de la vida. Esto representa una gran oportunidad para visualizar el escenario actual de la salud oral a partir de un análisis adecuado de los datos.

El bajo acceso a la atención odontológica es un problema de salud pública importante en la mayoría de los países en vías de desarrollo, y se acentúa más en grupos vulnerables y en edades extremas de la vida ${ }^{(10)}$. Lograr una adecuada cobertura de atención en servicios odontológicos es fundamental para la aplicación de medidas preventivas y tratamientos bucales. No obstante, los países con una marcada diversidad geográfica y socioeconómica presentan grandes desigualdades territoriales en los indicadores de salud oral de la población ${ }^{(11,12)}$. Concretamente, en Perú, el fragmentado sistema de salud empeora aún más la existencia de dichas desigualdades, por lo que es relevante mejorar el acceso a la atención odontológica para la utilización de las variadas estrategias costoefectivas existentes para la disminución de la

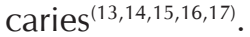

La mejora de la salud oral en niños peruanos dependería, en cierta medida, de un replanteamiento de los programas y políticas de salud que promueven el acceso a los servicios de salud oral de acuerdo al grupo de la población al que van dirigidos ${ }^{(18,19)}$. En ese sentido, conocer la distribución territorial de este problema de salud permitiría una mejor comprensión de los hechos y ayudaría a la toma de decisiones ${ }^{(20)}$, lo cual facilitaría la formulación e implementación de políticas públicas acordes a la realidad sanitaria nacional ${ }^{(21)}$

En la actualidad, los sistemas de información geográfica (SIG) se aplican a las investigaciones en salud con perspectiva espacial o territorial ${ }^{(22,23)}$. No obstante, en Perú, aún no se han desarrollado investigaciones relevantes en temas de salud oral que incorporen estas herramientas. Por estas razones, el objetivo del este estudio fue explorar los patrones de acceso a servicios de salud dental en menores de doce años en Perú.

\section{MATERIALES Y MÉTODOS}

\section{Diseño del estudio}

A partir de los datos de una encuesta nacional, se realizó un análisis que toma como área de estudio el total de las 25 regiones que integran el país para evaluar el acceso a los 
servicios de salud dental, al cual definimos como haber recibido al menos una atención odontológica en los últimos seis meses. Los resultados del análisis se representaron mediante mapas temáticos de cada una de las regiones agrupadas por cuantiles, con cada una de las variables estudiadas.

\section{Ámbito del estudio}

De acuerdo al Banco Mundial, la población peruana para el año 2014 era de aproximadamente 30 millones de habitantes y, para el 2013, el gasto total en salud bordeaba el $5,3 \%$ del producto bruto interno ${ }^{(24)}$. En Perú, luego de un proceso de descentralización, su territorio quedó dividido geopolíticamente en 25 regiones que se encuentran distribuidas en tres regiones naturales: la costa, con su vecindad al océano pacífico; la sierra, que se circunscribe a la cordillera de los Andes; y la selva, con su trópico (Figura 1).

Asimismo, el sistema de salud peruano se caracteriza por ser uno de los más fragmentados de América Latina, con cuatro subsistemas: el público, que subsidia los servicios de salud a la población de menores recursos y tiene establecimientos ubicados hasta en pequeñas comunidades; el sistema de seguridad social (EsSalud), que provee servicios a los trabajadores formales y sus dependientes, cuyos establecimientos más distantes están en capitales de provincias; el sector de sanidad de las fuerzas armadas y policiales; y el sector privado, para aquellos con disposición a pagar directamente por el servicio o a una aseguradora privada ${ }^{(25,26)}$. Con la creación del Seguro Integral de Salud y la promulgación de la Ley de Aseguramiento Universal, un $66 \%$ de la población está afiliada al seguro público de salud, y es atendida en los establecimientos de salud públicos bajo la rectoría del Ministerio de Salud (MINSA) ${ }^{(27)}$.

Según estimaciones del INEI, en Perú existen 6.952.948 de niñas y niños menores de doce años (3.545.273 de niñas y 3.407.675 de niños), de los cuales el 84,5\% se encuentra afiliado a algún seguro de salud, el $56,9 \%$ al Seguro Integral de Salud, el
$22,7 \%$ al seguro social de EsSalud y un 4,9\% a otro tipo de seguro ${ }^{(28)}$.

\section{Fuentes de información}

Como fuente de información para la elaboración del estudio se tomó la Encuesta Demográfica y de Salud Familiar - ENDES 2014 elaborada por el Instituto Nacional de Estadística e Informática (INEI). La encuesta se realizó en las 25 regiones del Perú y los resultados obtenidos tienen un nivel de representación regional. La muestra se caracteriza por ser bietápica, probabilística e independiente y autoponderada a nivel regional y por área urbana y rural. El tamaño muestral de la

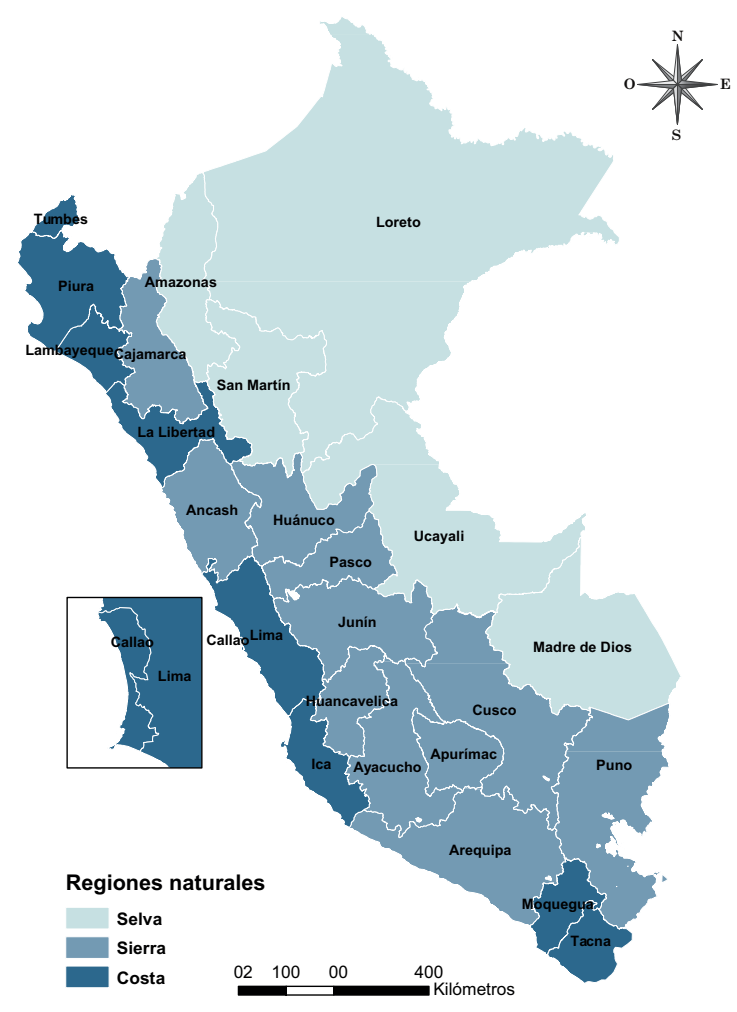

Figura 1. División geopolítica de Perú conformada por 25 regiones y su pertenencia a cada una de las tres regiones naturales.

Fuente: Elaboración propia basada en la cartografía regional del Ministerio del Ambiente del Perú (MINAM). 
ENDES 2014 fue de 29.806 viviendas, de las cuales 18.382 corresponden al área urbana y 11.424 al área rural, de modo que las estimaciones tienen representatividad a nivel nacional, urbano y rural, en las regiones naturales (costa, sierra y selva) y en cada una de las 25 regiones. La encuesta tiene dentro de sus poblaciones objetivo a los niños de 0 a 11 años, por lo cual, luego de excluir los datos faltantes, se incluyó una muestra de 25.285 menores de doce años residentes tanto en áreas urbanas como rurales de las 25 regiones que conforman el territorio peruano.

\section{Análisis de datos}

El diseño metodológico usado para el análisis de datos se dividió en dos fases. Un primer análisis de tipo cuantitativo, descriptivo y transversal de los módulos de la encuesta, obtenidos del portal web del INEI que se importaron al software estadístico Stata 14.1. Luego de la importación de la base de datos, se especificó el muestreo de la encuesta que incluía las ponderaciones según estratos, factores de expansión y efecto de diseño mediante el comando svy.

Las variables incluidas fueron: alguna vez recibió atención en servicio dental (qs803), tiempo desde la última atención en servicio dental (qs804c), edad (qs802d), regiones (hv024), lugar de atención (qs805) y lugar de residencia (hv025). Se creó la variable atención dental en los últimos seis meses, a partir de las variables alguna vez recibió atención en servicio dental y tiempo desde la última atención en servicio dental. Esta fase permitió el procesamiento de la encuesta para la obtención de frecuencias absolutas, medias y proporciones con intervalos de confianza del $95 \%$ para cada una de las categorías (grupos etarios, área de residencia, regiones naturales, lugar de atención y regiones) que fueron incluidas en una hoja de cálculo de Microsoft Excel ${ }^{\circledR}$, versión 2013.

La segunda fase consistió en un análisis exploratorio espacial de las proporciones regionales de menores con acceso a servicios de salud dental según regiones a nivel nacional, tipo de servicio de salud y lugar de residencia mediante layers en el software ArcGIS for Desktop versión 10.4. Para ello, se obtuvo del portal web del Ministerio del Ambiente (MINAM) de Perú la cartografía con los límites regionales en un archivo de tipo shapefile bajo el sistema de coordenadas WGS 1984 UTM Zone 17S. Con la finalidad de obtener una mejor visualización de los mapas temáticos, se agruparon las 25 regiones en cinco categorías según quintiles, siendo la categoría de color azul aquella con menor proporción de acceso.

\section{Consideraciones éticas}

El presente estudio no requirió la aprobación de un comité de ética por tratarse de un análisis secundario de una encuesta cuyos datos son de libre acceso y dominio público que no permite identificar a los menores participantes.

\section{RESULTADOS}

Del total de la muestra de menores de doce años considerada en la ENDES 2014, el $39,6 \%$ pertenecía al grupo etario de 0 a 4 años, el 40,6\% habitaba en la sierra y un $58,3 \%$ residía en zonas urbanas (Tabla 1 ).

El 26,7\% [IC 95\% $(26,2 ; 27,3)]$ tuvo acceso a los servicios de salud dental en los últimos seis meses. El acceso a la atención odontológica fue menor en los niños de 0 a 4 años de edad con el 18,3\% [IC 95\% (17,5; $19,0)]$ y en niños del área rural con el $22,3 \%$ [IC 95\% $(21,6 ; 23,2)]$. De los niños de 5 a 11 años el $32,3 \%$ tuvo acceso a servicios de salud dental (Tabla 1).

Las regiones de Huancavelica, Apurímac, Ayacucho, Pasco (todas ellas en la región andina central) y Lima (costa central) fueron las que tuvieron mayor acceso de menores de doce años a la atención en servicios odontológicos. La Tabla 1 muestra que el más alto porcentaje de población con acceso en el estudio fue del $45,9 \%$ en Huancavelica, y 
Tabla 1. Características generales de los menores de 12 años que accedieron a servicios de salud dental en los últimos seis meses $(n=25.285)$.

\begin{tabular}{|c|c|c|c|c|c|}
\hline \multirow[t]{2}{*}{ Características } & \multicolumn{2}{|c|}{$\begin{array}{l}\text { Número de menores } \\
\text { incluidos en la encuesta }\end{array}$} & \multicolumn{3}{|c|}{$\begin{array}{l}\text { Número y proporción de menores con } \\
\text { acceso a servicios dentales }\end{array}$} \\
\hline & $\mathbf{n}$ & $\%$ & $\mathbf{n}$ & $\%$ & IC $95 \%$ \\
\hline \multicolumn{6}{|l|}{ Grupos etarios } \\
\hline 0 - 4 años & 10.016 & 39,6 & 1.828 & 18,3 & 17,$5 ; 19,0$ \\
\hline 5 - 11 años & 15.269 & 60,4 & 4.934 & 32,3 & 31,$6 ; 33,1$ \\
\hline \multicolumn{6}{|l|}{ Área de residencia } \\
\hline Urbana & 14.739 & 58,3 & 4.405 & 29,9 & 29,$2 ; 30,6$ \\
\hline Rural & 10.546 & 41,7 & 2.357 & 22,3 & 21,$6 ; 23,2$ \\
\hline \multicolumn{6}{|l|}{ Región natural } \\
\hline Costa & 9.471 & 37,5 & 2.638 & 27,9 & 27,$0 ; 28,8$ \\
\hline Sierra & 10.267 & 40,6 & 3.089 & 30,1 & 29,$2 ; 31,0$ \\
\hline Selva & 5.547 & 21,9 & 1.035 & 18,7 & 17,$6 ; 19,7$ \\
\hline \multicolumn{6}{|c|}{ Institución de acceso $^{1}$} \\
\hline Ministerio de Salud & - & - & 3.681 & 54,4 & 53,$2 ; 55,6$ \\
\hline EsSalud & - & - & 1.068 & 15,8 & 14,$9 ; 16,7$ \\
\hline Privados & - & - & 1.820 & 26,9 & 25,$9 ; 28,0$ \\
\hline \multicolumn{6}{|l|}{ Regiones } \\
\hline Amazonas & 1.035 & 4,1 & 189 & 18,3 & 16,$0 ; 20,8$ \\
\hline Ancash & 1.145 & 4,5 & 224 & 19,6 & 17,$3 ; 22,0$ \\
\hline Apurímac & 899 & 3,6 & 378 & 42,0 & 38,$8 ; 45,4$ \\
\hline Arequipa & 794 & 3,1 & 250 & 31,5 & 28,$3 ; 34,8$ \\
\hline Ayacucho & 972 & 3,8 & 370 & 38,1 & 35,$0 ; 41,2$ \\
\hline Cajamarca & 963 & 3,8 & 215 & 22,3 & 19,$7 ; 25,1$ \\
\hline Callao & 783 & 3,1 & 240 & 30,7 & 27,$4 ; 34,0$ \\
\hline Cusco & 844 & 3,3 & 230 & 27,3 & 24,$3 ; 30,4$ \\
\hline Huancavelica & 850 & 3,4 & 390 & 45,9 & 42,$5 ; 49,3$ \\
\hline Huánuco & 993 & 3,9 & 270 & 27,2 & 24,$4 ; 30,1$ \\
\hline Ica & 922 & 3,6 & 276 & 29,9 & 27,$0 ; 33,0$ \\
\hline Junín & 840 & 3,3 & 234 & 27,9 & 24,$8 ; 31,0$ \\
\hline La Libertad & 970 & 3,8 & 220 & 22,7 & 20,$1 ; 25,4$ \\
\hline Lambayeque & 889 & 3,5 & 170 & 19,1 & 16,$6 ; 21,9$ \\
\hline Lima & 2.616 & 10,3 & 954 & 36,5 & 34,$6 ; 38,3$ \\
\hline Loreto & 1.364 & 5,4 & 224 & 16,4 & 14,$5 ; 18,5$ \\
\hline Madre de Dios & 939 & 3,7 & 258 & 27,5 & 24,$6 ; 30,5$ \\
\hline Moquegua & 609 & 2,4 & 188 & 30,9 & 27,$2 ; 34,7$ \\
\hline Pasco & 983 & 3,9 & 346 & 35,2 & 32,$2 ; 38,3$ \\
\hline Piura & 1.189 & 4,7 & 191 & 16,1 & 14,$0 ; 18,3$ \\
\hline Puno & 984 & 3,9 & 182 & 18,5 & 16,$1 ; 21,1$ \\
\hline San Martín & 1.016 & 4,0 & 203 & 20,0 & 17,$6 ; 22,6$ \\
\hline Tacna & 647 & 2,6 & 215 & 33,2 & 29,$6 ; 37,0$ \\
\hline Tumbes & 846 & 3,3 & 184 & 21,7 & 19,$0 ; 24,7$ \\
\hline Ucayali & 1.193 & 4,7 & 161 & 13,5 & 11,$6 ; 15,6$ \\
\hline
\end{tabular}


el más bajo fue del 13,5\% en Ucayali (en el sur de la selva). La distribución espacial del acceso a los servicios se muestra en la Figura 2. Se puede observar que de las 25 regiones que constituyen el territorio peruano, hubo un mayor acceso a la atención en servicios de salud dental en Huancavelica, Apurímac, Ayacucho y Pasco, que corresponden a la zona del centro de Perú. Asimismo, Lima, la región de la capital del país, tuvo un porcentaje de población con acceso del 36,5\% siendo la única región de la costa dentro del quintil de regiones con mayor acceso en nuestro estudio. Los porcentajes generales de población con acceso por regiones naturales fueron mayores en la costa y sierra peruana, con un $27,9 \%$ [IC $95 \%(27,0 ; 28,8)]$ y un $30,1 \%$ [IC 95\% $(29,2 ; 31,0)]$ respectivamente.

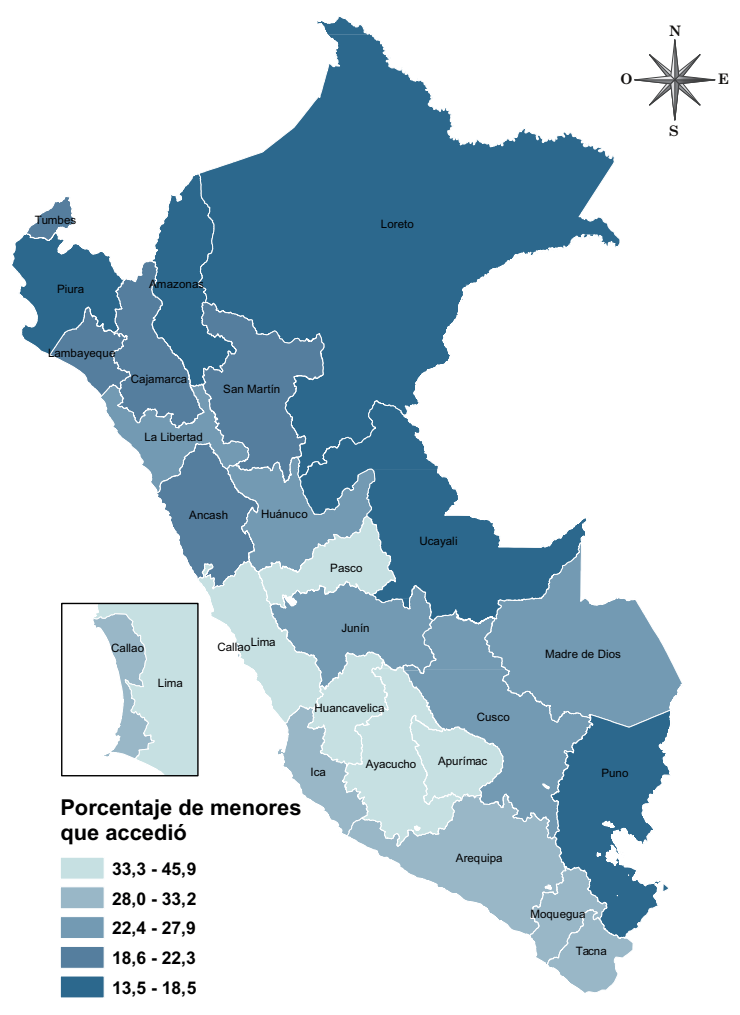

Figura 2. Porcentaje de menores de doce años que accedieron a servicios de salud oral según regiones. Perú, 2014.

Fuente: Elaboración propia basada en la Encuesta Demográfica y de Salud Familiar - ENDES 2014 y en la cartografía regional del Ministerio del Ambiente del Perú (MINAM).
A nivel de la costa, el mayor porcentaje poblacional con acceso se observó en Lima, Tacna, Arequipa, Moquegua y Callao. La selva fue la región que obtuvo el más bajo porcentaje de población con acceso a servicios odontológicos con el 18,7\% [IC 95\% $(17,6 ; 19,7)]$ (Tabla 1$)$.

En general los porcentajes de población de menores de doce años que tuvieron acceso a servicios odontológicos a través del MINSA, del EsSalud y de los servicios privados a nivel nacional fueron del $54,4 \%$ [IC 95\% (53,2; 55,6)], el 15,8\% [IC 95\% $(14,9 ; 16,7)$ ] y el $26,9 \%$ [IC $95 \%(25,9$; $28,0)$ ] respectivamente. Sin embargo, al estimar las tasas de acceso por tipo de servicios según regiones, la tasa más alta de acceso a la atención en servicios odontológicos del MINSA se halló en Huancavelica, Apurímac, Huánuco, Ayacucho y Cajamarca, y la más baja fue en Lambayeque, Lima, Ica, Arequipa y Moquegua. Con respecto a la atención a través del EsSalud, las regiones de Madre de Dios, Pasco, Moquegua, Lambayeque y Tumbes presentaron las más altas tasas de acceso, mientras que las más bajas corresponden a Huánuco, Huancavelica, Ayacucho, Apurímac y Puno. Las mayores tasas de acceso a la atención en servicios de salud oral privados corresponden a las regiones costeras de Lambayeque, La Libertad, Lima, Ica y Arequipa, mientras que Huancavelica, Apurímac, Pasco, Huánuco y Loreto poseen los menores valores (Figura 3).

La Figura 3 muestra que el menor porcentaje de población con acceso al MINSA es del $25,3 \%$, al EsSalud, del 5,9\% y a los servicios privados del 5,4\%, existiendo una brecha porcentual entre el MINSA y el EsSalud del $19,4 \%$, y del $19,9 \%$ entre el MINSA y los servicios privados. Además se aprecia una diferencia del $0,5 \%$ entre el EsSalud y los servicios privados. Los mayores porcentajes de población de menores de 12 años que accedieron a los servicios de salud oral fueron: para el MINSA, el 88,7\%; el EsSalud, $33,6 \%$; y los servicios privados, el $54 \%$, con diferencias porcentuales del $55,1 \%$ entre el MINSA y el EsSalud, y del $34,7 \%$ entre el MINSA y los servicios privados. 

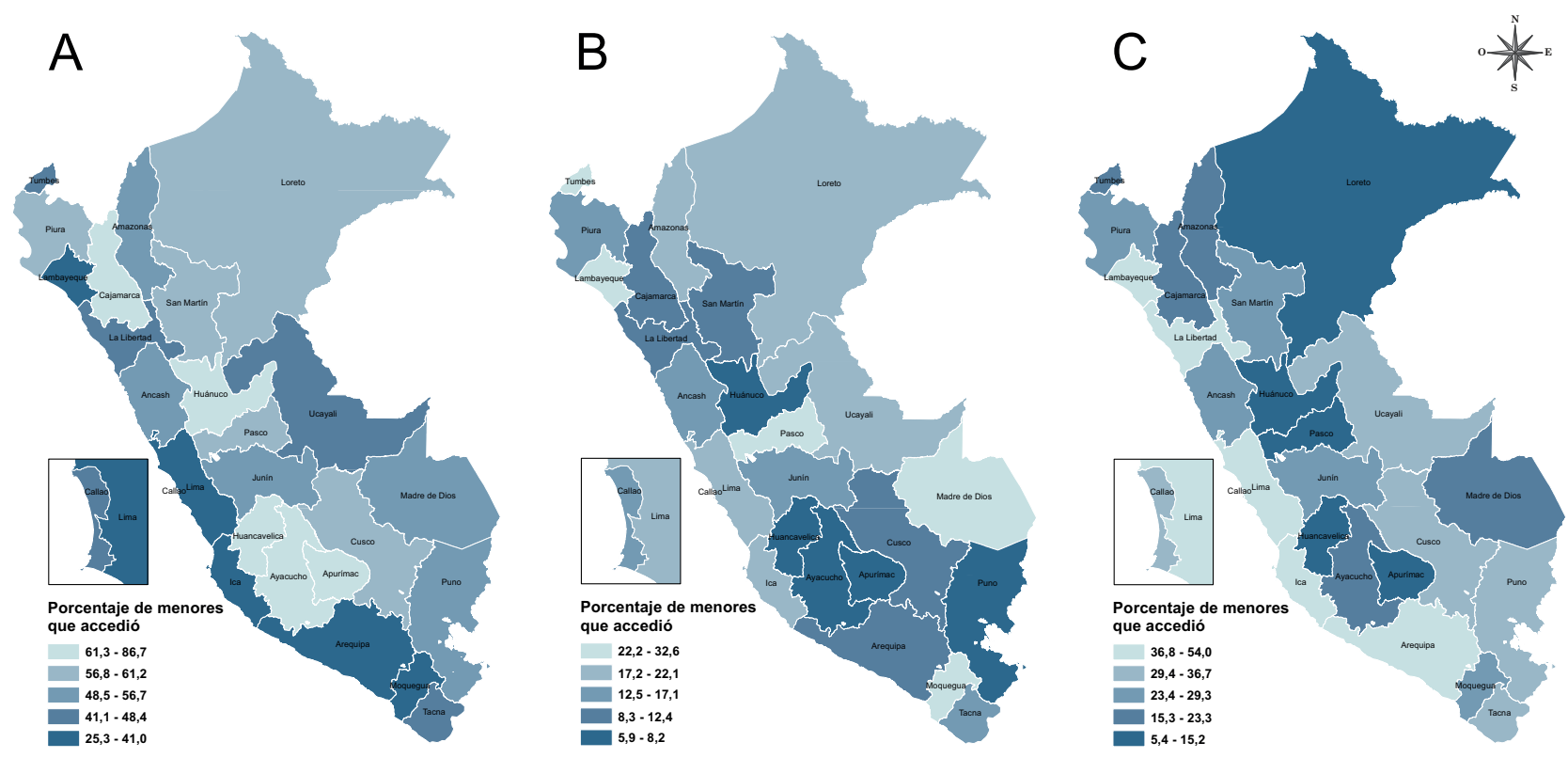

Figura 3. Porcentaje de menores de doce años que accedieron a servicios de salud oral, según tipo de servicio y regiones. Perú, 2014.

Fuente: Elaboración propia basada en la Encuesta Demográfica y de Salud Familiar - ENDES 2014 y en la cartografía regional del Ministerio del Ambiente del Perú (MINAM).

A. Ministerio de Salud (públicos)

B. EsSalud (seguridad social)

C. Servicios privados
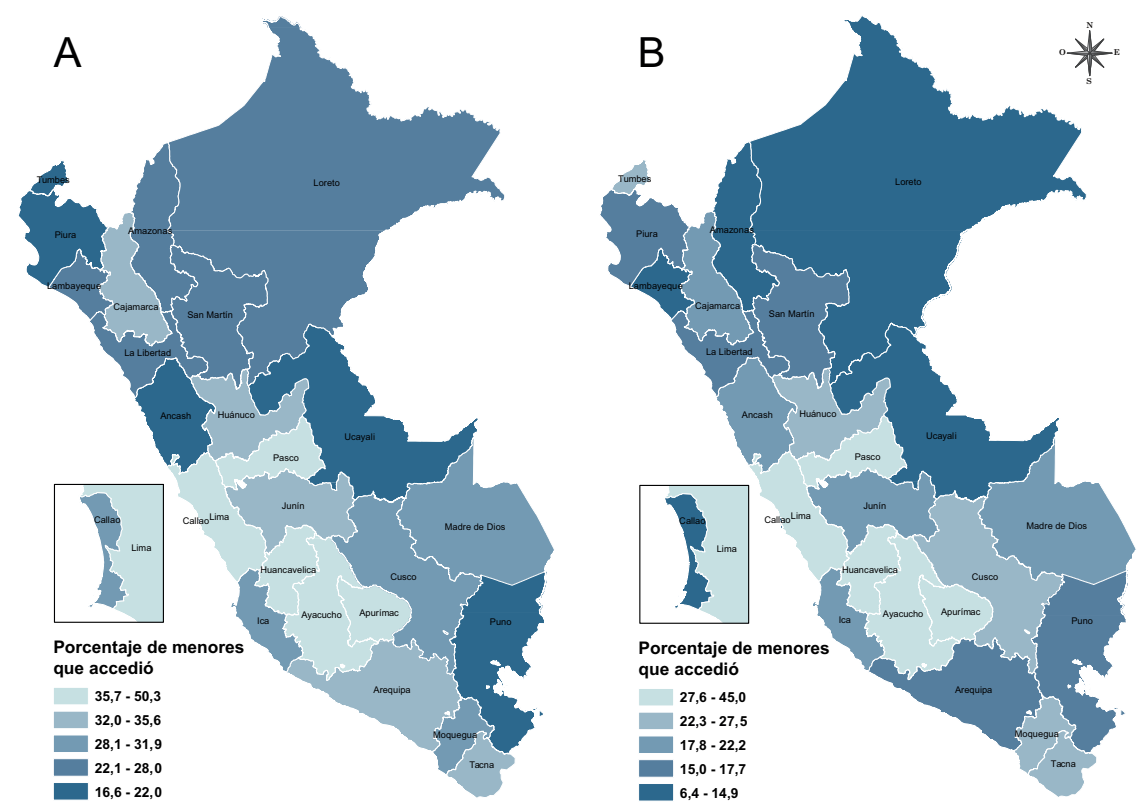

Figura 4. Porcentaje de menores de doce años que accedieron a servicios de salud oral, según área de residencia y regiones. Perú, 2014.

Fuente: Elaboración propia basada en la Encuesta Demográfica y de Salud Familiar - ENDES 2014 y en la cartografía regional del Ministerio del Ambiente del Perú (MINAM).

A. Área urbana.

B. Área rural. 
Las regiones de Huancavelica, Ayacucho, Apurímac, Pasco y Lima mostraron los más altos índices de acceso a la atención en servicios odontológicos, tanto en la zona urbana como rural. Las zonas urbanas pertenecientes a Puno, Tumbes, Ancash, Ucayali y Piura obtuvieron los menores valores de acceso a la atención odontológica, al igual que las zonas rurales de Piura, Amazonas, Ucayali, Lambayeque y Loreto (Figura 4).

En la Figura 4 se puede observar que los menores porcentajes de población con acceso en las zonas rurales y urbanas de Perú son del $6,4 \%$ y del $16,6 \%$ respectivamente, existiendo una diferencia porcentual del $10,2 \%$ entre estos valores; mientras que los mayores porcentajes poblacionales de acceso se encuentran en la zona rural con un $45 \%$ y la urbana con un $50,3 \%$ lo que muestra una diferencia del $5,3 \%$. Cabe precisar que la región Callao es la única región en Perú que no cuenta con población rural.

\section{DISCUSIÓN}

El presente estudio identificó un bajo porcentaje de niños menores de doce años que accedió a la atención en servicios odontológicos en los últimos seis meses, así como también, una marcada heterogeneidad interregional en el acceso. Pueden ser múltiples los factores que modelan los patrones del acceso a los servicios de salud dental en menores, tales como factores culturales del niño y sus padres, lugar de vivienda urbana o rural, factores socioeconómicos, distancia a los centros de salud, la disposición y distribución de profesionales odontólogos en una determinada área geográfica, o la presencia o no de programas sociales que incentiven el uso de los servicios de salud ${ }^{(29,30)}$. Además, se conoce que dichos factores pueden variar, entre ubicaciones geográficas y grupos demográficos, en un mismo país ${ }^{(31,32)}$. Siendo así, es importante estudiar todos estos factores, partiendo de la identificación de la situación actual y las variaciones que pueden acontecer dentro de un territorio.
Existen diversas formas de definir y operacionalizar el concepto de acceso a los servicios de salud. Algunos autores se centran en las características de la población, mientras otros lo hacen desde el punto de vista de la oferta de los servicios de salud; sin embargo, se han planteado combinaciones de ambas perspectivas. Asimismo, hay diferencias en cuanto al alcance del concepto de acceso. Existe, por un lado, un dominio acotado que toma en cuenta el proceso desde la búsqueda hasta el inicio de atención y que, a su vez, identifica factores de facilitan o dificultan la obtención del acceso y, por otro, un dominio amplio que agrega las necesidades de salud, la percepción y diferencias de las necesidades, el deseo de atención y el proceso de toma de decisiones como antecedentes a la búsqueda de atención, considerando en algunos casos la continuidad y los resultados del contacto inicial ${ }^{(33)}$. En nuestro estudio, la variable acceso fue construida sobre la base de la respuesta de las personas entrevistadas en la ENDES que habían recibido atención en un servicio dental o por un odontólogo y el tiempo transcurrido desde la última atención en algún establecimiento del sector público o privado en Perú, sin profundizar en otros aspectos asociados.

El panorama hallado en nuestro estudio es similar al que reportó Chile en un estudio a nivel nacional en el año 2003, en el que solo un $12,8 \%$ de los niños menores de 5 años y un 30,9\% de los niños entre 6 y 14 años tuvieron acceso a atención dental en los seis meses previos a su participación en ese estudio $^{(34)}$. No obstante, las tasas de atención de países desarrollados se muestran muy distantes de la peruana. Por ejemplo, un estudio realizado en el Reino Unido en el año 2013, mostró que el $87,0 \%$ de los niños de 5 años, el $92,0 \%$ de los niños de 8 años y el $90,0 \%$ de los niños de 12 años de edad reportaron haber tenido acceso a los servicios de salud dental durante el año previo a la realización de la encuesta ${ }^{(35)}$.

El hecho de que solo un $18,3 \%$ de los niños del Perú del grupo etario de 0 a 4 años haya tenido acceso a servicios de salud dental en los últimos seis meses Ilama la atención, 
pues la recomendación de la AAPD es realizar al menos una visita al odontólogo en dicho periodo de tiempo ${ }^{(6)}$. Por otra parte, la presencia de caries en este grupo etario es un factor predictor de aparición de esta enfermedad en la dentición permanente ${ }^{(36)}$, la cual inicia su proceso eruptivo entre los seis y siete años de edad, por lo que la atención antes, durante y después de esa transición resulta importante. Parece ser que los padres aún piensan que las visitas odontológicas de sus hijos de corta edad son innecesarias o poco importantes, debido a que se producirá un recambio de la dentición primaria a la permanente, y solo los llevan a recibir atención odontológica una vez que existe dolor, abscesos u otras complicaciones de la caries dental ${ }^{(37)}$. No obstante, estas conductas pueden condicionar negativamente la salud oral de estos niños cuando tengan mayor $\operatorname{edad}^{(38)}$.

En el grupo etario de 5 a 11 años, se observó un mayor porcentaje de población con acceso que en los menores de 5 años de edad. Sin embargo, sigue siendo escaso, pues la mayor parte del recambio dentario se desarrolla en este periodo, y es necesario prevenir la aparición de caries acudiendo al odontólogo. De igual manera, es en esta etapa que se debe iniciar el modelamiento de hábitos con respecto a la salud oral, para lo cual la orientación por parte del profesional es primordial. Además, la evidencia muestra que la presencia de caries a los 12 años de edad representa un importante predictor de riesgo de padecer caries a los 18 años $^{(39)}$, lo que podría contribuir al incremento de caries dental en edades adultas en aquellos niños menores de 12 años con la enfermedad y sin acceso a servicios de salud dental.

La prevalencia de caries dental en niños en Perú y en el resto de los países podría ser menor, pues existen métodos que han demostrado gran efectividad a través de los años (por ejemplo, la aplicación tópica de flúor por el profesional de salud, el uso de sellantes de fosas y fisuras). Y es un hecho que tanto la promoción y prevención, como la atención oportuna en los niños de este grupo de edad podrían ofrecer grandes beneficios para la salud oral y general ${ }^{(40)}$. La aplicación de estos métodos preventivos podría evitar la pérdida dentaria prematura e infecciones odontológicas que, la mayoría de veces, ocasionan alteraciones de la erupción dentaria, dolor y maloclusiones, incrementando el riesgo de caries, enfermedad periodontal y otros problemas relacionados. Un mayor acceso a la atención de la salud dental podría aumentar el uso de medidas preventivas costo-efectivas para caries dental en niños; y lograr ese aumento depende de una reformulación de las actuales políticas de salud pública que promueven el acceso, pues nuestros resultados indican una deficiencia en este aspecto ${ }^{(41,42)}$.

Cabe mencionar que se han implementado políticas y programas para mejorar el uso de servicios de salud en el territorio peruano, tal es el caso del Aseguramiento Universal de Salud (AUS) y el programa "JUNTOS". Este último funciona bajo la modalidad de un programa de transferencias condicionadas que se inició en zonas andinas con altos porcentajes de pobreza y extrema pobreza, brindando un incentivo monetario sobre la base de la asistencia de sus afiliados a programas de salud, nutrición, educación e identidad. Al respecto, algunos estudios consideran que el uso de los servicios de salud, dentro del marco del programa "JUNTOS", presenta ligeros aumentos porcentuales con el pasar de los años(43). De hecho, las regiones con mayor antigüedad del programa -Huancavelica, Apurímac, Ayacucho y Huánuco ${ }^{(44)}$ - tuvieron los más altos porcentajes de población con acceso a servicios de atención odontológica en escenarios rurales y urbanos, que incluso llegan a ser mayores que los de la capital peruana. Además, es importante resaltar que las regiones de la sierra tienen un mayor acceso a establecimientos públicos del MINSA y de EsSalud, siendo muy bajo en el sector privado. Por el contrario, en Lima predomina el acceso al sector privado, siendo mínimo en las instituciones públicas de salud.

Dentro de las medidas para asegurar el acceso a los servicios de salud, si bien el AUS puede favorecer a que las personas tengan mayores posibilidades de acceso al eliminar 
la barrera económica, los resultados principales de este estudio no reflejan buenos porcentajes de población que haya accedido a servicios odontológicos. El AUS pareciera ser una de las soluciones al problema del acceso a la salud dental y, por ende, a la gran prevalencia de caries dental. Sin embargo, este no garantiza un efecto positivo sobre las inequidades/desigualdades en la experiencia de caries dental ${ }^{(19)}$, ni en el acceso a la atención odontológica, pues existen otros factores relacionados que podrían influir negativamente ${ }^{(11)}$, y que deberían ser tomados en cuenta al formular políticas y programas de salud. Por ejemplo, las regiones de Loreto, Piura y Ucayali que tenían entre el $60 \%$ y el $80 \%$ de su población asegurada en el año 2011, tuvieron los menores porcentajes de acceso en nuestro estudio, a diferencia de Huancavelica, Ayacucho y Apurímac con similar tasa de aseguramiento pero mucho mayores porcentajes de población con acceso según los hallazgos del estudio ${ }^{(45)}$.

Se encontró, además, que las regiones de la selva poseen los más bajos niveles de acceso a los servicios de salud oral, e índices muy altos de caries a nivel nacional(4). Paradójicamente, en un reciente estudio de las enfermedades más prevalentes en la Amazonía peruana, la caries dental no fue considerada como una de ellas ${ }^{(46)}$. Asimismo, según lo hallado en el presente estudio, el caso del acceso a la atención odontológica se podría considerar como un problema en esta área geográfica, pues las regiones Amazonas, Loreto y Ucayali mostraron los porcentajes poblacionales de acceso más bajos del Perú. Como posibles explicaciones a estos resultados, se puede decir que ciertas costumbres pertenecientes a minorías étnicas, propias de las regiones de la sierra y la selva peruana, podrían estar contribuyendo a mantener malos hábitos en salud en estos grupos de población ${ }^{(47)}$. Entre ellas podrían mencionarse el desconocimiento o el desinterés por buscar atención dental, el uso de remedios caseros, una oferta de servicios de atención dental y de profesionales odontólogos insuficiente en estas regiones para cubrir las necesidades de salud oral de esta fracción de la población. Por citar un ejemplo, en Ucayali se reportaron 40 odontólogos del MINSA en el año $2013^{(48)}$ para una población de aproximadamente 432.000 habitantes, cifras que distan mucho del número de odontólogos recomendados por la Organización Mundial de la Salud (OMS) para ese tamaño de población. Más aún, considerando que los establecimientos del MINSA están presentes en lugares más alejados y de difícil acceso en comparación con los de EsSalud y los del sector privado y que, en ese escenario, la capacidad resolutiva odontológica, en caso de contar con profesionales, es aún insuficiente por problemas de dotación de insumos y/o recursos odontológicos.

No es novedad que América Latina se encuentra caracterizada por grandes disparidades entre los sectores urbanos y rurales como las que hemos podido evidenciar en esta investigación. A pesar del esfuerzo de los programas sociales en Perú, estos problemas aún persisten. Pareciera ser que el acceso a la salud dental predomina en las zonas urbanas, como se puede observar en las diferencias porcentuales. Sin embargo, en algunas zonas de Perú se observan porcentajes de atención en las zonas rurales que se acercan a los de las zonas urbanas. Se han reportado estudios en otros contextos que establecen que el hecho de vivir en zonas rurales y alejadas de los servicios de salud dental, no parece actuar como barrera para la búsqueda de tratamiento ${ }^{(49)}$. En términos generales, no se conocen en profundidad los factores que causan esta tendencia; pero, en el caso de Perú, se podría deducir que los programas que dan facilidades de acceso a la salud estarían actuando positivamente sobre la demanda de atención odontológica en ciertas regiones, por lo que se deberían evaluar los factores detrás de ese éxito, para intentar replicarlos en las regiones de menor acceso.

El panorama que presenta esta investigación en niños menores de doce años en Perú expone cómo las medidas de salud pública llevadas a cabo sobre salud oral no conllevan a un acceso adecuado de la población infantil a nivel nacional. Por lo tanto, es 
prioritario replantear las estrategias actuales de cobertura y acceso a la atención en salud oral, priorizando por regiones. El uso de los SIG puede ayudar a tener una visión general de la ocurrencia del acceso a la atención odontológica a nivel nacional, generando evidencia que facilite la toma de decisiones; con ello se puede empezar a implementar intervenciones destinadas a mejorar la situación actual. Asimismo, permite comparar escenarios futuros con la realidad actual como línea de base, y orientar futuras investigaciones que complementen y profundicen los datos obtenidos en el presente estudio como, por ejemplo, estudios que permitan conocer los factores que operan detrás del bajo acceso a la atención en servicios odontológicos de las diferentes regiones, pues podrían no ser semejantes, permitiendo diseñar e implementar intervenciones a medida de las necesidades.

Vale señalar que este estudio tiene ciertas limitaciones que son propias de los estudios realizados con datos secundarios. Pudieron haberse cometido errores en el llenado de las encuestas y es posible que los informantes no brindaran los datos correctos acerca de la atención recibida y su relación de temporalidad. Algunos de los encuestados pudieron haber confundido a los encuestadores del
INEI con personal del programa JUNTOS y haber brindado datos incorrectos, por desconocimiento o miedo a perder el aseguramiento de salud o los incentivos económicos. Sin embargo, la importancia de este estudio radica, en parte, en la realización del análisis de los datos de la ENDES, que es de alcance nacional y nos asegura un nivel de representatividad regional. Un análisis interesante a explorar es el estudio de los factores de acceso a los servicios dentales que podrían explicar las diferencias y los patrones encontrados en las regiones y permitiría integrar variables como disponibilidad de profesionales, e índices socioeconómicos, entre otros, que podrían generar desigualdades geográficas.

En conclusión, el acceso en Perú a los servicios de salud dental para la población menor de doce años es bajo y concentra sus mayores porcentajes de población con acceso en las regiones andinas, y los menores porcentajes en la selva. Además, la zona urbana de Perú también tuvo mayor concentración de acceso que la rural. La observación de la distribución espacial del acceso a los servicios dentales permitió identificar y agrupar regiones según patrones espaciales comunes, para enfocar acciones en materia de salud pública.

\section{REFERENCIAS BIBLIOGRÁFICAS}

1. Bagramian RA, Garcia-Godoy F, Volpe AR. The global increase in dental caries: A pending public health crisis. American Journal of Dentistry. 2009;22(1):3-8.

2. Çolak H, Dülgergil ÇT, Dalli M, Hamidi MM. Early childhood caries update: A review of causes, diagnoses, and treatments. Journal of Natural Science, Biology, and Medicine. 2013;4(1):29-38.

3. Jin LJ, Lamster IB, Greenspan JS, Pitts NB, Scully C, Warnakulasuriya S. Global burden of oral diseases: emerging concepts, management and interplay with systemic health. Oral Diseases. 2015. doi: 10.1111/odi.12428.

4. Ministerio de Salud. Prevalencia nacional de caries dental, fluorosis del esmalte y urgencia de tratamiento en escolares de 6 a 8, 10, 12 y 15 años,
Perú 2001-2002 [Internet]. Lima: Oficina General de Epidemiología, MINSA; 2005 [citado 25 feb 2016]. Disponible en: http://goo.gl/x6prdM.

5. Petersen PE, Bourgeois D, Ogawa H, Estupinan-Day S, Ndiaye C. The global burden of oral diseases and risks to oral health. Bulletin of the World Health Organization. 2005;83:661-669.

6. American Academy of Pediatric Dentistry. Guideline on periodicity of examination, preventive dental services, anticipatory guidance/counseling, and oral treatment for infants, children, and adolescents [Internet]. 2013 [citado 25 feb 2016]. Disponible en: http://goo.gl/HPYLZX.

7. Ramos-Gomez FJ, Crystal YO, Ng MW, Crall J, Featherstone JD. Pediatric dental care: prevention and management protocols based on caries risk assessment. Journal of the California Dental Association. 2010;38(10):746-761. 
8. Alarcón M, Aquino C, Quintanilla C, Raymundo L, Álvarez J. Odontología basada en evidencia: las 82 revistas de mayor impacto. International Journal of Odontostomatology. 2015;9(1):43-52.

9. Pulgar R, Jimenez-Fernandez I, Jimenez-Contreras E, Torres-Salinas D, Lucena-Martin C. Trends in world dental research: an overview of the last three decades using the Web of Science. Clinical Oral Investigations. 2013;17(7):1773-1783.

10. Hernandez-Vasquez A, Vilcarromero S, Rubilar-Gonzalez J. Neglect of oral health in children as a public health problem in Peru. Revista Peruana de Medicina Experimental y Salud Pública. 2015;32(3):604-605.

11. Edelstein $\mathrm{BL}$, Chinn $\mathrm{CH}$. Update on disparities in oral health and access to dental care for America's children. Academic Pediatrics. 2009;9(6):415-419.

12. Seerig LM, Nascimento GG, Peres MA, Horta $\mathrm{BL}$, Demarco FF. Tooth loss in adults and income: Systematic review and meta-analysis. Journal of Dentistry. 2015;43(9):1051-1059.

13. Marinho VC, Higgins JP, Sheiham A, Logan S. Fluoride toothpastes for preventing dental caries in children and adolescents. The Cochrane Database of Systematic Reviews. 2003(1):CD002278.

14. Ahovuo-Saloranta A, Hiiri A, Nordblad A, Makela $M$, Worthington $H V$. Pit and fissure sealants for preventing dental decay in the permanent teeth of children and adolescents. The Cochrane Database of Systematic Reviews. 2008(4):CD001830.

15. Iheozor-Ejiofor Z, Worthington HV, Walsh T, O'Malley L, Clarkson JE, Macey R, et al. Water fluoridation for the prevention of dental caries. The Cochrane Database of Systematic Reviews. 2015;6:CD010856.

16. Marinho VC, Higgins JP, Logan S, Sheiham A. Fluoride gels for preventing dental caries in children and adolescents. The Cochrane Database of Systematic Reviews. 2002(2):CD002280.

17. Nakre PD, Harikiran AG. Effectiveness of oral health education programs: A systematic review. Journal of International Society of Preventive \& Community Dentistry. 2013;3(2):103-115.

18. Guay AH. Access to dental care: solving the problem for underserved populations. Journal of the American Dental Association. 2004;135(11): 1599-1605.
19. Ismail AI, Sohn W. The impact of universal access to dental care on disparities in caries experience in children. Journal of the American Dental Association. 2001;132(3):295-303.

20. Delamater PL, Messina JP, Shortridge AM, Grady SC. Measuring geographic access to health care: raster and network-based methods. International Journal of Health Geographics. 2012;11(1):15.

21. Instituto Nacional de Estadística e Informática. Perú: enfermedades no transmisibles y transmisibles [Internet]. Lima: INEI; 2013 [citado 25 feb 2016]. Disponible en: https://goo.gl/aPfD8p.

22. Loyola E, Castillo-Salgado C, Najera-Aguilar P, Vidaurre M, Mujica OJ, Martinez-Piedra R. Geographic information systems as a tool for monitoring health inequalities. Revista Panamericana de Salud Pública. 2002;12(6):415-428.

23. Musa GJ, Chiang PH, Sylk T, Bavley R, Keating W, Lakew B, et al. Use of GIS Mapping as a Public Health Tool-From Cholera to Cancer. Health Services Insights. 2013;6:111-116.

24. World Bank. World Development Indicators [Internet]. 2013 [citado 20 feb 2016]. Disponible en: http://data.worldbank.org/country.

25. Sanchez-Moreno F. The national health system in Peru. Revista Peruana de Medicina Experimental y Salud Pública. 2014;31(4):747-753.

26. Alcalde-Rabanal JE, Lazo-Gonzalez O, Nigenda G. The health system of Peru. Salud Pública de México. 2011;53(Suppl 2):S243-S254.

27. Ypanaque-Luyo $P$, Martins $M$. Utilization of outpatient health services in the Peruvian population. Revista Peruana de Medicina Experimental y Salud Pública. 2015;32(3):464-470.

28. Instituto Nacional de Estadística e Informática. En el Perú existen alrededor de 7 millones de niñas y niños menores de 12 años de edad [Internet]. Lima: INEI; 2015 [citado 4 jun 2016]. Disponible en: https://goo.gl/ZbXSU6.

29. Patrick DL, Lee RSY, Nucci M, Grembowski D, Jolles CZ, Milgrom P. Reducing oral health disparities: A focus on social and cultural determinants. BMC Oral Health. 2006;6(Suppl 1):S4.

30. Mouradian WE, Wehr E, Crall JJ. Disparities in children's oral health and access to dental care. Jama. 2000;284(20):2625-2631.

31. Heaton LJ, Smith TA, Raybould TP. Factors influencing use of dental services in rural and urban communities: considerations for practitioners in 
underserved areas. Journal of Dental Education. 2004;68(10):1081-1089.

32. Fisher-Owena SA, Soobader MJ, Gansky SA, Isong IA, Weintraub JA, Platt LJ, Newacheck PW. Geography matters: state-level variation in children's oral health care access and oral health status. Public Health. 2016;134:54-63.

33. Ballesteros MS, Freidin B. Reflections on the conceptualization and measurement of access to health services in Argentina: The case of the National Survey of Risk Factors 2009. Salud Colectiva. 2015;11(4):523-535.

34. Delgado $\mathrm{BI}$, Cornejo-Ovalle $\mathrm{M}$, Jadue $\mathrm{H} \mathrm{L}$, Huberman J. Determinantes sociales y de equidad de acceso a la salud dental en Chile. Científica Dental. 2013;10(2):101-109.

35. Tsakos G, Hill K, Chadwick B, Anderson T. Children's dental health survey 2013, Report 1: Attitudes, behaviours and children's dental health: England, Wales and Northern Ireland [Internet]. 2015 [citado 21 mar 2016]. Disponible en: http:// goo.gl/NzkDdR.

36. Li Y, Wang W. Predicting caries in permanent teeth from caries in primary teeth: an eight-year cohort study. Journal of Dental Research. 2002;81(8):561-566.

37. Lourenço CB, Saintrain MVL, Vieira A. Child, neglect and oral health. BMC Pediatrics. 2013;13:188.

38. Tagliaferro EP, Pereira AC, Meneghim Mde C, Ambrosano GM. Assessment of dental caries predictors in a seven-year longitudinal study. Journal of Public Health Dentistry. 2006;66(3):169-173.

39. David J, Raadal M, Wang NJ, Strand GV. Caries increment and prediction from 12 to 18 years of age: a follow-up study. European Archives of Paediatric Dentistry. 2006;7(1):31-37.

40. Finucane D. Rationale for restoration of carious primary teeth: a review. European Archives of Paediatric Dentistry. 2012;13(6):281-292.
41. World Health Organization. Oral health, strategies for oral disease prevention and health promotion [Internet]. Geneva: WHO [citado 21 mar 2016]. Disponible en: http://goo.gl/VqlcPq.

42. Rowan-Legg A. Oral health care for children - a call for action. Paediatrics \& Child Health. 2013;18(1):37-43.

43. Presidencia del Consejo de Ministros. Informe compilatorio: "El Programa JUNTOS, resultados y retos" [Internet]. Lima: Presidencia del Consejo de Ministros; 2010 [citado 16 feb 2016]. Disponible en: http://goo.gl/J7LIFJ.

44. Ministerio de Desarrollo e Inclusión Social. Juntos en cifras, 2005-2014 [Internet]. Lima: MIDIS; 2014 [citado 16 feb 2016]. Disponible en: http://goo.gl/9psqai.

45. Ministerio de Salud. Análisis de situación de salud del Perú [Internet]. Lima: MINSA; 2013 [citado 16 feb 2016]. Disponible en: http://goo. gl/34W8bp.

46. Guimarães MGS, Braña AM, Oliart-Guzmán $H$, Branco $F$, Delfino BM, Pereira TM, et al. Child health in the Peruvian Amazon: Prevalence and factors associated with referred morbidity and health care access in the city of Iñapari. Journal of Tropical Medicine. 2015. doi: 10.1155/2015/157430.

47. Malqvist M, Hoa DT, Liem NT, Thorson A, Thomsen S. Ethnic minority health in Vietnam: a review exposing horizontal inequity. Global Health Action. 2013;6:1-19.

48. Ministerio de Salud. Perfil epidemiológico de salud bucal en el Perú [Internet]. Lima: MINSA; 2013 [citado 25 feb 2016]. Disponible en: https:// goo.gl/dEsCbW.

49. McKernan SC. Geographic accessibility and utilization of orthodontic services among Medicaid children and adolescents. Journal of Public Health Dentistry. 2013;73(1):56-64. utilizada con finalidades comerciales, a menos que se obtenga el permiso. 